\title{
Miradas epistemológicas desde distintas perspectivas teóricas sobre la discapacidad
}

\section{Epistemological Views from Different Theoretical Perspectives of Disability}

Recibido 01 de febrero de 2012 • Corregido 22 de octubre de 2012 • Aceptado 07 de noviembre de 2012

Resumen. Este artículo presenta distintas perspectivas teóricas y sus aproximaciones epistemológicas en el estudio de la discapacidad. Explica distintos enfoques para concebir la discapacidad según los diferentes aspectos que cada uno enfatiza, así como algunos de los debates entre dichos enfoques. El artículo aporta a la clarificación teórico-conceptual para el estudio e investigación sobre la discapacidad.

Palabras claves. Perspectivas teóricas, enfoques, aproximaciones epistemológicas, modelos, discapacidad.

Abstract. This paper provides theoretical perspectives and the corresponding epistemological approaches on the study of disabilities. It explains diverse focuses to see disabilities from their different points of view; it also includes debates between those focuses. This paper contributes to a conceptual-theoretical clarification for the study and research on disabilities.

Keywords. Theoretical perspectives, approaches, epistemological approaches, models, disabilities.

\footnotetext{
Máster en Currículum e Instrucción con énfasis en Educación Especial Bilingüe de The George Washington University, USA. Doctoranda del Programa Latinoamericano de Doctorado en Educación, Universidad de Costa Rica. Académica de la División de Educación Básica del Centro de Investigación y Docencia en Educación (CIDE), Universidad Nacional, Costa Rica donde ha formado parte del Proyecto UNA educación de calidad que atiende a estudiantes con discapacidad en dicha institución. Tiene publicaciones en los campos de Pedagogía y de Educación Especial.
} 
URL: http://www.una.ac.cr/educare

\section{A manera de introducción}

Tanto en las ciencias de la salud como en las ciencias sociales, se ha abordado la discapacidad como tema de estudio desde distintas perspectivas. En las ciencias de la salud, sobre todo a partir de la segunda mitad del siglo XIX, se adopta una visión médica y terapéutica que enfatiza el déficit, el tratamiento, la curación y la rehabilitación de las distintas discapacidades. En las ciencias sociales, durante el siglo XX y lo que va del siglo XXI, la discapacidad cobra interés para la investigación desde los campos de la psicología, la educación y la sociología (Oliver, 2002). Su propósito es explicar cómo las personas sin discapacidad perciben a las personas con discapacidad, y cómo esto influye en el trato social y en la inserción de estas en distintos espacios educativos, laborales, sociales y políticos.

\section{Perspectivas teóricas en el estudio de la discapacidad}

En las ciencias sociales, la temática de la discapacidad se ha investigado desde distintas perspectivas teóricas de las cuales Vargas (2010) identifica las cuatro siguientes: positivista, cognitiva, interpretativa y sociocrítica, como se explica a continuación y se ilustra en la Tabla 1.

a) Perspectiva positivista: en el campo de la psicología, la investigación sobre discapacidad de corte científico positivista cobra auge en la primera mitad del siglo XX, principalmente en Estados Unidos. La perspectiva positivista se focaliza en características objetivas en términos de conductas observables y medibles.

b) Perspectiva cognitiva: se desarrolla en contraposición al conductismo a partir de la segunda mitad del siglo XX, tanto en Estados Unidos como en Europa. Esta perspectiva se centra en estudiar y explicar las funciones y procesos cognitivos.

c) Perspectiva interpretativa: tiene origen en la psicología experimental de Wundt (Farr, 1994), luego adquiere un carácter social al conjugarse con la sociología europea de Durkheim (Farr, 1994). Posteriormente, al integrar aspectos referentes a la construcción colectiva de significados, deriva en el enfoque del interaccionismo simbólico y, más recientemente, en la teoría de las representaciones sociales.

d) Perspectiva sociocrítica: se desarrolla desde la sociología; sostiene que las relaciones de poder están determinadas por las bases estructurales de la sociedad (Barnes, 2003; Oliver, 2002); influencia posturas pedagógicas, psicológicas, políticas y sociales. 
Tabla 1

Perspectivas teóricas, aproximaciones epistemológicas y miradas a la discapacidad

\begin{tabular}{|c|c|c|}
\hline Perspectiva teórica & Aproximación epistemológica & Mirada a la discapacidad \\
\hline Positivista & $\begin{array}{l}\text { Asume que existe una verdad objetiva, observables y } \\
\text { medible. }\end{array}$ & $\begin{array}{l}\text { Rasgos deficitarios } \\
\text { atributos individuales }\end{array}$ \\
\hline Cognitiva & $\begin{array}{l}\text { Asume que el significado es construido con base en } \\
\text { procesos cognitivos perceptuales y sociales. }\end{array}$ & $\begin{array}{l}\text { Percepciones, actitudes, } \\
\text { estereotipos y prejuicios }\end{array}$ \\
\hline Interpretativa & $\begin{array}{l}\text { Asume que no existe una verdad objetiva, y por tanto, los } \\
\text { sentidos y significados se construyen socialmente según la } \\
\text { interpretación que se haga de la realidad. }\end{array}$ & Construcciones sociales \\
\hline Sociocrítica & $\begin{array}{l}\text { Asume que el sujeto es actuante en un determinado } \\
\text { marco histórico-cultural donde las élites dominantes } \\
\text { procuran perpetuar patrones sociales de dominación y } \\
\text { opresión; presupone relaciones de poder en relación con } \\
\text { la acumulación del capital y las bases estructurales de la } \\
\text { sociedad. }\end{array}$ & $\begin{array}{l}\text { Relaciones opresivas } \\
\text { de poder }\end{array}$ \\
\hline
\end{tabular}

Nota: Elaboración propia a partir de investigación documental

Cada una de las perspectivas teóricas señaladas se caracteriza y diferencia por los supuestos que asume en su aproximación epistemológica, entendida esta como la forma de acercarse a la realidad que se conoce y a la relación que en ese acercamiento se establece entre la persona cognoscente, el objeto del conocimiento y el conocimiento mismo. Las distintas perspectivas teóricas continúan aún vigentes dependiendo de la posición epistemológica que se asuma en la investigación y de la aproximación a la realidad investigada.

A partir de las distintas perspectivas teóricas identificadas, se originan cinco enfoques conceptuales los cuales, al abordar el tema de la discapacidad, ofrecen distintas miradas, como se explica a continuación.

\section{Distintas miradas a la discapacidad desde las diferentes perspectivas teóricas}

\section{a. La mirada de los atributos individuales}

El estudio de la discapacidad adquiere una mirada reduccionista al focalizarse en las características individuales y atribuir la condición de discapacidad a la persona en términos deficitarios. Desde esta mirada se tipifican y categorizan las características. Además, se concibe la formación de actitudes hacia las personas con discapacidad sobre una base conductual reactiva ante atributos individuales, según lo expresa la siguiente definición de Allport (1935) sobre la actitud: 
URL: http://www.una.ac.cr/educare

Una disposición mental y neurológica, que se organiza a partir de la experiencia que ejerce una influencia directriz o dinámica sobre las reacciones del individuo respecto de todos los objetos y a todas las situaciones que les corresponden. (p.152)

\section{b. La mirada de las percepciones}

Desde el enfoque cognitivo, se han estudiado los efectos perceptuales que producen las características manifiestas de la discapacidad en las personas sin discapacidad. Autoras como Wright (1983) indican que, al ver una persona con alguna discapacidad, la atención se centra, perceptualmente, en la discapacidad y no en la persona y, la impresión visual provoca una actitud negativa. Este argumento sostiene que se tiende a identificar y categorizar a la persona por su apariencia, en este caso, por la manifestación visible de su discapacidad. Este enfoque explica que lo que se percibe como diferente se rechaza; lo no familiar provoca emociones negativas o resistencia; la proximidad con lo no familiar produce un efecto perceptual que genera juicios, pre-concepciones, como también, actitudes negativas.

\section{c. La mirada de las actitudes, los estereotipos y los prejuicios}

El estudio de las actitudes cobra importancia dentro de la psicología social ya que gran parte de las conductas sociales de los individuos se manifiestan como actitudes hacia las demás personas. Dado que las actitudes y los prejuicios se originan en procesos de interacción con otras personas, se denominan 'actitudes sociales'.

Según Rodríguez (1991), las actitudes están conformadas por un componente afectivo, otro cognoscitivo y otro conductual, lo cual quiere decir que están compuestas por un sentimiento, una creencia o idea y se expresan en un comportamiento o acción. Se puede establecer una relación entre prejuicio, estereotipo y actitud, ya que el prejuicio es el componente afectivo de la actitud; el estereotipo, el componente cognitivo y la actitud, la expresión conductual de ambos. En esta línea, se entiende que la actitud es una manifestación conductual, es decir, implica un comportamiento, mientras que los prejuicios y los estereotipos no necesariamente se manifiestan en comportamientos, si bien pueden provocarlos. Sin embargo, para otras autoras como Fiske (1998), los estereotipos son un componente cognoscitivo de las actitudes; los prejuicios, el componente afectivo; pero la manifestación conductual es la discriminación.

Para otros autores como Devine (1995), el estereotipo refleja una construcción social y cultural. Los prejuicios se relacionan con los estereotipos, ya que los estereotipos son formas de tipología social potencialmente engañosas que no admiten variabilidad (Nolan, 2003); consisten en creencias que se tienen acerca de un grupo, por lo general minoritario; al aplicarlos individualmente a las personas que pertenecen a ese grupo, se convierten en prejuicios (Fiske, 2004). Así, los prejuicios son una expresión de afectividad negativa en términos individuales que los estereotipos expresan en términos sociales. 
Los prejuicios hacia las personas con discapacidad, como hacia la discapacidad misma, se han basado en creencias tales como que la discapacidad produce infelicidad y sufrimiento, o que las personas con discapacidad son incapaces de aceptarla y llevar una vida como la de cualquier otra persona. De estas creencias se derivan muchas actitudes negativas que han sido medidas con escalas actitudinales. Sin embargo, según Devine (1995), las nuevas conceptualizaciones sobre los prejuicios surgen de las posiciones hacia el racismo simbólico moderno en Estados Unidos. El racismo simbólico alude en específicamente a prejuicios raciales $y$, en general, parte de la evidente incongruencia entre las respuestas manifiestas y las respuestas no manifiestas que no logran medir las escalas de actitudes.

\section{d. La mirada de las construcciones sociales}

Desde la perspectiva interpretativa, se identifica la mirada de las construcciones sociales y desde esta se identifican dos líneas. Por un lado, el enfoque del interaccionismo simbólico y, por otro lado, el de las representaciones sociales.

El enfoque interaccionista explica que socialmente se otorgan significados, según la interpretación que se haga de la realidad. Este enfoque sostiene que las personas se construyen mutuamente y construyen significados en conjunto, en la interacción con los demás, por lo que los significados tienen un carácter social. Así, para Ibáñez (1989), lo social, los significados y la intersubjetividad, se construyen en la interacción entre las personas. Según lbáñez (1989), "nada es social si no es instituido en el mundo de significados comunes propios de una colectividad de seres humanos, es decir, en el marco y por medio de la intersubjetividad" (p. 227). Por tanto, la interacción es una condición para generar lo social; la construcción de significados es un proceso complejo en el que intervienen el lenguaje, la comunicación, lo contextual y otros factores que encuentran interpretación en los elementos simbólicos de las estructuras sociales. Al respecto, cabe mencionar los aportes de Goffman (1981) al estudiar los comportamientos e interacciones sociales y analizar los procesos de estigmatización, entendiendo por estigmatización la situación de rechazo y descalificación que vive cualquier persona que no goza de aceptación social. Las investigaciones sobre discapacidad en esta línea analizan cómo al etiquetar socialmente a las personas con discapacidad, se les estigmatiza y, por tanto, se desvaloriza su identidad. Ello incide en su vida, dadas las implicaciones sociales, educativas, políticas y legales, entre otras, según lo defina la construcción social que se hace de esa identidad y que se legitime socialmente. En este sentido, cabe mencionar los trabajos de historia de vida de Hunt (1966) y Morris (1991), quienes, desde su propia experiencia, relatan cómo la sociedad excluye y aísla a las personas con deficiencias físicas y las convierte en un grupo estigmatizado.

La mirada de las representaciones sociales explica que nos representamos a las personas y las situaciones en un imaginario colectivo; este comprende las relaciones sociales e individuales a partir de las relaciones del sujeto con el mundo y consigo mismo. Desde esta mirada, las personas con discapacidad están representadas históricamente con una connotación negativa 
URL: http://www.una.ac.cr/educare

en el imaginario colectivo. Las investigaciones sobre discapacidad que adoptan este enfoque parten de la teoría postulada por Moscovici (1981), quien define las representaciones sociales en términos de sistemas de creencias propios del sentido común.

\section{e. La mirada sociocrítica}

Desde la mirada sociocrítica, autores como Finkelstein (1980) sostienen que la discapacidad refleja una relación social opresiva entre la gente con deficiencias físicas y la sociedad. Concuerdan con esta posición Ferrante y Ferreira (2008), quienes retoman las propuestas teóricas de Bourdieu (1991) sobre las relaciones de dominación y de poder de la clase dominante sobre un grupo minoritario oprimido. Desde este enfoque, el modelo social británico sostiene que "las personas con discapacidad son un grupo social oprimido"2 (Shakespeare y Watson, 2002, p. 12). Autores como Ferreira (2007) atribuyen a las ciencias médicas un reduccionismo funcionalista, al pretender reducir la discapacidad a algo fisiológico y; a la psicología, por proponer su tratamiento sin considerar las dimensiones contextuales sociales en relación con las barreras que afectan el desenvolvimiento de las personas en el entorno.

\section{Debates teóricos en el estudio de la discapacidad}

A partir de las distintas miradas presentadas, se identifica una línea de debate sobre el tema de las actitudes hacia las personas con discapacidad, dado que una corriente emerge desde el enfoque conductista, con los aportes de Allport (1935), y otra desde la psicología cognitiva, con los aportes de Heider (1944).

Según Jaspars y Fraser (1984), Allport (1935) incorporó al campo de la psicología estadounidense el concepto actitudes en el sentido de atributos personales, en momentos en que, en Estados Unidos, el comportamiento social no se estudiaba en términos conductuales, sino en términos de percepción, debido a la influencia de la psicología alemana y austríaca de la Gestalt. Esto sucede en pleno auge del positivismo estadounidense, por lo que las investigaciones se desarrollan en la línea positivista, con la aplicación de instrumentos de investigación para medir las actitudes, tales como las escalas tipo Likert en los años treinta y las de Guttman en los cuarenta.

Asimismo, desde el campo de la rehabilitación y con un enfoque cognitivo, Wright (1983), en la línea de la Gestalt traída de Austria a Estados Unidos por Heider (1944), estudió las actitudes hacia la discapacidad como consecuencia de las percepciones y propuso el concepto negative bias ${ }^{3}$ para referirse a la causa de las actitudes negativas. Este aporte de Wright (1983), que se introduce en los informes de investigación y en los textos estadounidense de los

\footnotetext{
Cita textual traducida por la autora desde el original.

Sesgo negativo.
} 
años sesenta, ha quedado pendiente de investigar, pues no se ha retomado a pesar de las posibilidades investigativas actuales desde el campo las neurociencias.

Otro debate teórico que se identifica se refiere al estudio de las actitudes desde la psicología social y desde las representaciones sociales. Sin embargo, hay un punto de encuentro entre estos enfoques, que se remonta a su punto de origen común en Alemania. De acuerdo con Jaspars y Fraser (1984), las propuestas de Wundt a la psicología hacían referencia a las representaciones colectivas y a la necesidad de una psicología social. De igual forma, las propuestas sociológicas de Durkheim ya hablaban de la diferencia entre las representaciones individuales y las colectivas.

Las ideas de Durkheim las introducen en Estados Unidos los precursores del interaccionismo simbólico: el filósofo Mead y el sociólogo Thomas, de la escuela de Chicago en los años veinte (Farr, 1994). Según Farr (1994), después de haber estudiado con Wundt en Alemania, Thomas utiliza el concepto actitudes sociales para "definir la psicología social como el estudio de las actitudes sociales" (Farr, 1994, p. 31). En el contexto europeo, Moscovici (1981) se inspira en los trabajos de Durkheim para desarrollar su teoría sobre las representaciones sociales.

\section{Modelos teóricos sobre discapacidad}

Las distintas perspectivas teóricas y sus respectivos enfoques enmarcan distintos modelos sobre la discapacidad que discrepan en sus conceptualizaciones; se diferencian en la visión que se tiene de la discapacidad y en las implicaciones sociales que generan. La noción de modelo se refiere a representaciones conceptuales y relacionales utilizadas para explicar y comprender la realidad de cierta manera, ya que perfilan aspectos relevantes a una problemática de estudio. Con este entendido, los modelos proporcionan marcos referentes para hacer investigación y para quienes la utilizan o recurren a ella.

Autores como Devenney (2005) y Finkelstein (1980) mencionan tres modelos teóricos de la discapacidad, a saber: el modelo médico o rehabilitador, el modelo de la caridad y el modelo social. Otros autores seleccionan cuatro modelos, por ejemplo, Altman (2001) selecciona el modelo de Nagi (1965, citado por Altman, 2001), el modelo de los institutos de medicina (IOM), que a su vez parte del de Nagi (Brandt y Pope, 1997 citados por Altman 2001), y los dos modelos aportados por la Organización Mundial de la Salud en los años 1980 y 1999. Por su parte, Lou Royo (2011) identifica cuatro modelos: el enfoque médico-biológico, el modelo bio-psico-social, el modelo universal y el modelo social. Otros autores, como Barnes (2007), especifican solo dos modelos: el modelo individual y el modelo social, dadas las discrepancias sustanciales entre ambos, razón por la que se mencionan a continuación.

\footnotetext{
Cita textual traducida por la autora desde el original.
} 
El modelo individual concibe una dimensión exclusivamente personal y parte de la visión de la discapacidad como una característica que se le atribuye a la persona en términos deficitarios, es decir, como algo extraño de lo considerado normal. Este modelo, impulsado desde el campo de la medicina, logra impactar en la psicología y la educación, se asocia con segregación e institucionalización, con prácticas médicas rehabilitadoras y con prácticas educativas en espacios aparte.

Por otro lado, el modelo social tiene implicaciones ideológicas, políticas y culturales (Barnes, 2003), al concebir la discapacidad en una dimensión relacional, la cual define términos de interacción de la persona con el entorno. Este modelo fue impulsado por activistas de la Union of the Physically Impaired Against Segregation ${ }^{5}$ (UPIAS) del Reino Unido en los años setenta. Su auge se debe a que sus proponentes han logrado impactar en políticas y legislación nacional, así como en acuerdos internacionales en materia de discapacidad. Este movimiento logra credibilidad académica desde la sociología crítica, gracias a Finkelstein (1980), Oliver (1990; 1996); Shakespeare (1993) y Barnes (2007). Además, logra que se escuche la voz de las personas con discapacidad, reconoce las luchas sociales de los movimientos de las personas con discapacidad (Oliver, 2002) y promueve la investigación emancipadora.

De acuerdo con Lou (2011), algunos autores como Palacios y Romañach (2006, citados por Lou, 2011) abogan por trascender el modelo social, pues critican que este modelo persiste en enfatizar y diferenciar la capacidad/discapacidad como condiciones opuestas. Por lo que, proponen optar por el modelo de la diversidad, cuya posición teórica es universalista, puesto que hace énfasis en la dignidad humana. Desde esta perspectiva, el principio de universalización consiste en que todas las personas viven con el riesgo de presentar alguna discapacidad durante su vida, lo cual justifica la importancia de legislar tomando en cuenta dicho principio. Dichos autores acotan el término personas con diversidad funcional para referirse a las personas con discapacidad, término que es impulsado por el Movimiento por una vida independiente (Lou, 2011), el cual surge en Estados Unidos en la década de 1970 y promulga el derecho de las personas con discapacidad a asumir el control de sus vidas, ejercer sus decisiones y, con ello, superar el proteccionismo asistencialista.

\section{Reflexiones finales}

A partir de las perspectivas teóricas positivista, cognitiva, interpretativa y crítica desde las cuales se ha estudiado e investigado el tema de la discapacidad, se identifican distintos enfoques que se caracterizan por los aspectos que cada uno enfatiza.

Así, la perspectiva positivista enfatiza los atributos individuales, caracteriza las causas de la discapacidad como condición individual, justifica el tratamiento y la rehabilitación. El enfoque cognitivo destaca, por un lado las percepciones y, por otro, las actitudes, los estereotipos y prejuicios hacia las personas con discapacidad. La perspectiva interpretativa sostiene que la discapacidad es una construcción social y, por tanto, subjetiva. Esta perspectiva enmarca

Unión de Impedidos Físicamente contra la Segregación 
la mirada de las representaciones sociales y del interaccionismo simbólico. Por último, la perspectiva crítica perfila la mirada sociocrítica, la cual concibe la discapacidad como producto de una relación de poder opresiva la cual evidencia que históricamente las personas con discapacidad han sido segregadas de la fuerza laboral.

La identificación de enfoques y modelos a partir de las distintas perspectivas teóricas que los enmarcan tiene implicaciones importantes para la investigación sobre discapacidad, puesto que posibilitan clarificar el posicionamiento epistemológico en términos de aproximación al conocimiento, al hacer investigación y, consecuentemente, en el diseño metodológico, la selección de técnicas y estrategias de investigación que se utilicen. Lo anterior tiene relevancia, siempre y cuando derive en aplicaciones prácticas en beneficio de las personas con discapacidad, y del colectivo social en general, para la convivencia, la participación humana y el respeto a la diversidad.

Las distintas miradas epistemológicas desde las perspectivas teóricas analizadas invitan a un posicionamiento epistemológico social y políticamente comprometido. Posicionamiento que promueva el cambio de la realidad socio-cultural para superar modelos y formas obsoletas de mirar la discapacidad y, así, propiciar el desarrollo de la autonomía personal y la vida independiente de las personas con discapacidad.

\section{Referencias}

Allport, G. (1935). Attitudes [Actitudes]. En C. Murchison (Ed.), A Handbook of Social Psychology [Manual de psicología social] (151-153). Massachusetts: Clark University Press.

Altman, B. (2001). Disability Definitions, Models, Classification Schemes, and Applications [Definiciones de discapacidad, modelos, esquemas de clasificación y aplicaciones]. En G. L. Albrecht, K. D. Seelman y M. Bury (Eds.), Handbook of Disability Studies [Manual de estudios de discapacidad] (pp. 97-122). London: Sage.

Barnes, C. (2003). Independent Living, Politics and Policy in the United Kingdom: A Social Model Account [Vida independiente, política y políticas en el Reino Unido: Un recuento del modelo social]. Review of Disability Studies, 1(4), 5-13.

Barnes, C. (enero, 2007). Disability, higher education and the inclusive society [Discapacidad, educación superior y la sociedad inclusiva]. British Journal of Sociology of Education, 28(1), 135-145.

Bourdieu, P. (1991). El sentido práctico. Madrid: Taurus.

Devenney, M. J. V. (2005). The Social Representations of Disability: Fears, Fantasies and Facts [Las representaciones sociales de la discapacidad: Miedos, fantasías y hechos]. (Tesis doctoral). Clare College, Cambridge University.

Devine, P. (1995). Prejudice and outgroup perception [Prejuicio y percepción del exogrupo]. En A. Tesser (Ed.), Advanced Social Psychology [Psicología social avanzada] (pp. 467-526). New York: McGraw Hill. 
URL: http://www.una.ac.cr/educare

Farr, R. (1994). Attitudes, Social Representations and Social Attitudes. [Actitudes, representaciones sociales y actitudes sociales]. Papers on Social Representations, 3(1), 30-33.

Ferrante, C. y Ferreira, M. A. (2008). Cuerpo, discapacidad y trayectorias sociales: Dos estudios de caso comparados. Revista de Antropología Experimental, 8(29), 403-428. Recuperado de http://www.ujaen.es/huesped/rae/articulos2008/29ferrante08.pdf

Ferreira, M. A. V. (octubre, 2007). Sociología de la discapacidad: Investigación y compromiso. Ponencia presentada en el III congreso nacional discapacidad y universidad, Zaragoza. Recuperado de http://www.um.es/discatif/TEORIA/Ferreira Zaragoza.pdf

Finkelstein, V. (1980). Attitudes and Disabled People: Issues for Discussion [Actitudes y personas con discapacidad: Hechos para la discusión]. Washington, DC: World Rehabilitation Fund. Recuperado de http://www.leeds.ac.uk/disability-studies/archiveuk/finkelstein/attitudes. pdf

Fiske, S. (1998). Stereotyping, prejudice and discrimination. [Estereotipos, prejuicio y discriminación]. En D. Gilbert, S. Fiske y G. Lindzey (Eds.), Handbook of social psychology [Manual de psicología social]. (4ª. Ed.). New Your: Mc Grow Hill.

Fiske, S. (2004). Social Beings. Core Motives in Social Psychology [Seres sociales: Motivos centrales en psicología social]. USA, NY: Wiley \& Sons.

Goffman, E. (1981). Forms of talk [Formas de hablar]. Philadelpha: University of Pennsylvannia Press.

Heider, F. (noviembre, 1944). Social Perception and Phenomenal Causality [Percepción social y causalidad fenomenal]. Psychology Review, 51(6), 358-374.

Hunt, P. (1966). Estigma: La experiencia de la discapacidad. Londres: Geoffry Chapman.

Ibáñez, T. (1989). La psicología social como dispositivo desconstruccionista. En T. Ibánez (Ed.), El conocimiento de la realidad social. (pp. 217-243). Barcelona: Sendai.

Jaspars, J. y Fraser, C. (1984). Attitudes and Social Representations [Actitudes y representaciones sociales]. En R. M. Farr y S. Moscovici, S. (Eds.), Social Representations [Representaciones sociales] (pp. 101-124). Cambridge: Cambridge University Press.

Lou, M. (2011). Discapacidad: Concepto y modelos explicativos. En: M. Lou (Ed.), Atención a las necesidades educativas específicas (pp. 19-38). Madrid: Ediciones Pirámide.

Morris, J. (1991). Pride against Prejudice: transforming attitudes to disability [Orgullo contra prejuicio: Transformando actitudes hacia la discapacidad]. London: The Women's Press.

Moscovici, S. (1981). On Social Representation [Sobre representación social]. En J. P. Forgas (Comp.), Social Cognition. Perspectives in Everyday Life [Cognición social. Perspectivas en la vida diaria]. Londres: Academic Press. 
Nolan, P. (octubre, 2003). Learning to Live Together [Aprendiendo a vivir juntos]. Adults Learning, 15(2), 12-14.

Oliver, M. (1990). The Politics of Disablement. Critical Texts in Social Work and the Welfare State [Las políticas de discapacidad. Textos críticos en trabajo social y el estado de bienestar]. Londres: MacMillan Press.

Oliver, M. (1996). Defining Impairment and Disability: Issues at Stake [Definiendo deficiencia y discapacidad: Hechos en juego]. En C. Barnes y G. Mercer (Eds.). Exploring the Divide: IIIness and Disability [Explorando lo dividido: Enfermedad y discapacidad] (pp. 29-54). Leeds: The Disability Press.

Oliver, M. (diciembre, 2002). Emancipatory Research: A Vehicle for Social Transformation or Policy Development [Investigación emancipatoria: Un vehículo para la transformación social o el desarrollo de políticas]. Trabajo presentado en el $1^{\text {st }}$ Annual Disability Research Seminar, Dublin. Recuperado de http://www.leeds.ac.uk/disability-studies/archiveuk/ Oliver/Mike's\%20paper.pdf

Rodríguez, A. (1991). Psicología social. México, D.F.: Editorial Trillas.

Shakespeare, T. (1993). Disabled People's Self-Organisation: A new social movement? [Organización propia de las personas con discapacidad: ¿Un nuevo movimiento social?]. Disability, Handicap and Society, 8(3), 249-264. Recuperado de http://www.um.es/discatif/ PROYECTO DISCATIF/Textos discapacidad/00 Shakespeare.pdf

Shakespeare, T. y Watson, N. (2002). The social model of disability: an outdated ideology? [EI modelo social de discapacidad: una ideología obsoleta?] Research in Social Science and Disability, 2, 9-28.

Vargas, M. (2010). Estado del arte: Perspectivas teóricas y metodológicas en la investigación sobre discapacidad. Programa Latinoamericano de Doctorado en Educación. Facultad de Educación. Universidad de Costa Rica. Manuscrito en preparación.

Wright, B. A. (1983). Physical Disability, a Psychosocial Approach [Discapacidad física, un enfoque psicosocial] ( $2^{\text {a }}$ ed.). NY: Harper \& Row Publishers.

\section{Cómo citar este artículo, según APA:}

Vargas, M. C. (2012). Miradas epistemológicas desde distintas perspectivas teóricas sobre la discapacidad. Revista Electrónica Educare, 16(3), 145-155. Consultado de http://www.revistas.una.ac.cr/index.php/EDUCARE/issue/current

Nota: Para citar este artículo en otros sistemas puede consultar el hipervínculo "Como citar el artículo" en la barra derecha de nuestro sitio web:

http://www.revistas.una.ac.cr/index.php/EDUCARE/index 